\title{
Interfaces of ZnO Nanowires Grown on Semiconducting Surfaces
}

\author{
Joysurya Basu, R. Divakar, Julia Deneen, Xinyu Wang, ${ }^{*}$ Heiko O. Jacobs, ${ }^{*}$ C. Barry \\ Carter \\ Department of Chemical Engineering and Materials Science, University of Minnesota, \\ 421 Washington Ave SE, Minneapolis, MN 55455, USA \\ * Department of Electrical and Computer Engineering, University of Minnesota, 200 \\ Union St SE, Minneapolis, MN 55455, USA
}

The large direct band gap of $\mathrm{ZnO}$ makes it an important semiconductor. $\mathrm{ZnO}$ can be grown in the form of nanowires on various substrates, which makes it a potential candidate for nanoparticle-based device applications such as heterojunction solar cells. Aligned nanowires of $\mathrm{ZnO}$ can be grown on semiconductor surfaces to make $p-n$ junctions. The characteristics and performance of devices are critically dependant on the alignment, distribution and the nanowire-substrate interface character. While various methods to control the alignment and distribution of $\mathrm{ZnO}$ nanowire on different surfaces have been reported in the literature [1], the structure of the interface between the $\mathrm{ZnO}$ nanowire and the semiconducting surface is almost unknown. The motivation of this work is to characterize the interface of $\mathrm{ZnO}$ nanowires grown on a $\mathrm{Si}$ surface.

$\mathrm{ZnO}$ crystals were grown on a $\mathrm{Si}$ surface by thermal oxidation of zinc acetate. $\mathrm{ZnO}$ nanowires were subsequently grown using hydrothermal synthesis [2]. They were characterized using a FEI Tecnai G2 F30 TEM operated at $300 \mathrm{kV}$. The nanowires were prepared for TEM by wedge cleaving the substrate. HRTEM study of the interface was carried out on specimens prepared by conventional cross-section techniques.

Using this method, fairly dense and aligned nanowires grow on the Si surface. The nanowires are typically $100 \mathrm{~nm}$ in radius and about $1 \mu \mathrm{m}$ long. Occasionally defects along the length of the nanowires can be observed. The interface between the nanowires and the Si substrate is interesting. It consists of three layers. Between the nanowires and the Si substrate a 30-40 nm-thick amorphous layer can be observed. While the interface between the nanowire and the amorphous layer is fairly flat and straight, the interface between the amorphous layer and the Si surface is not so well defined (Figure 1).

The nanowire growth on the $\mathrm{Si}$ substrate can be explained by the transport and dehydroxylization of the $\mathrm{Zn}(\mathrm{OH})_{\mathrm{n}}$ at the $\mathrm{Si}$ surface. The high density of the $\mathrm{ZnO}$ nanocrystals can be explained by the fact that $\mathrm{ZnO}$ nanowires can be grown without any assistance from $\mathrm{ZnO}$ nanocrystals. The amorphous layer is responsible for the current through the junction being about four orders of magnitude smaller than expected [3]. It is thought to be generated during the heat treatment used to generate $\mathrm{ZnO}$ seed nanocrystals on the Si substrate. The hexamethylenetetraamine used for the hydrothermal synthesis is also a strong oxidizer. It is believed that oxidation starts on the $\mathrm{Si}$ surface and the oxide interface moves into the $\mathrm{Si}$ substrate, which is why the interface between $\mathrm{Si}$ and the amorphous oxide layer is not flat, while the $\mathrm{ZnO}$ nanowires grow on the amorphous 
oxide layer. The present study reports characterization of such nanowire-substrate interfaces. [4].

\section{References}

[1] J. Grabowska et al., J. Mater. Sci.: Materials in Electronics, 16 (2005) 397.

[2] L. E. Greene et al., Angew. Chem. Int. Ed., 42 (2003) 3031.

[3] X. Wang and H. Jacobs, unpublished research, 2006

[4] This research is supported by the University of Minnesota through the MRSEC Program of the National Science Foundation under Award \# DMR-0212302.
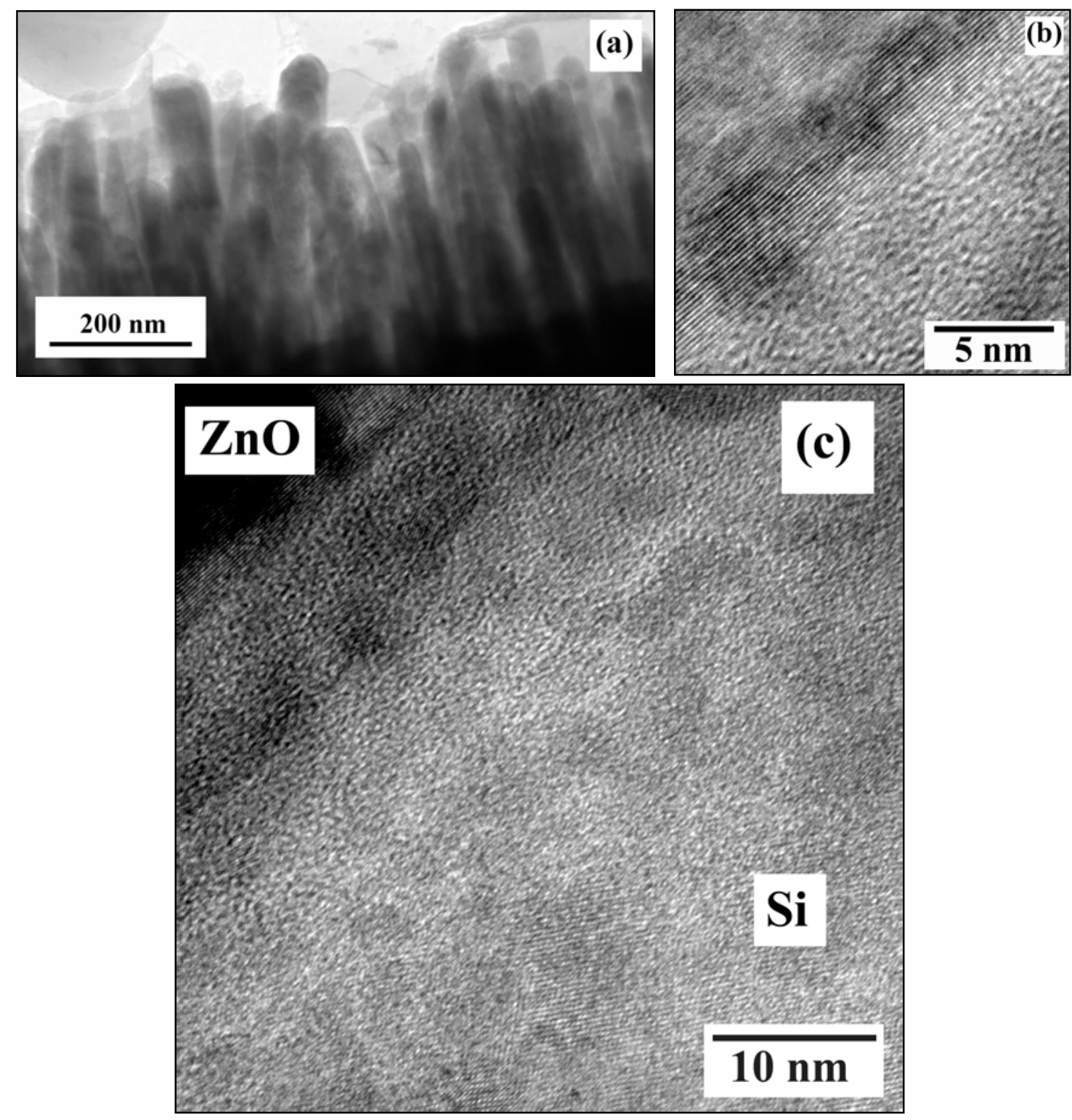

FIG. 1. (a) Overview of $\mathrm{ZnO}$ nanowires on a Si substrate, (b) Lattice image at the base of a nanowire. The lattice planes of the nanowire correspond to $\{10 \overline{1} 2\}$, (c) HRTEM image showing the $\mathrm{ZnO}$ nanowire (top left) and the Si substrate (bottom right) separated by an amorphous region. 